\title{
The effect of length scale on the determination of geometrically necessary dislocations via EBSD continuum dislocation microscopy
}

\author{
T.J. Ruggles ${ }^{\mathrm{a}, \mathrm{b}, *}$, T.M. Rampton ${ }^{\mathrm{c}}$, A. Khosravani ${ }^{\mathrm{d}}$, D.T. Fullwood ${ }^{\mathrm{b}}$ \\ ${ }^{a}$ National Institute of Aerospace, 100 Exploration Way, Hampton, VA 23666, USA \\ ${ }^{b}$ Department of Mechanical Engineering, Brigham Young University, Provo, UT 84602, USA \\ ${ }^{c}$ EDAX Inc., 91 McKee Drive, Mahwah, NJ 07430, USA \\ ${ }^{d}$ Woodruff School of Mechanical Engineering, Georgia Institute of Technology, Atlanta, GA 30332, USA
}

\begin{abstract}
Electron backscatter diffraction (EBSD) dislocation microscopy is an important, emerging field in metals characterization. Currently, calculation of geometrically necessary dislocation (GND) density is problematic because it has been shown to depend on the step size of the EBSD scan used to investigate the sample. This paper models the change in calculated GND density as a function of step size statistically. The model provides selection criteria for EBSD step size as well as an estimate of the total dislocation content. Evaluation of a heterogeneously deformed tantalum specimen is used to asses the method.
\end{abstract}

Keywords: High resolution EBSD, continuum dislocation microscopy, EBSD step size, dislocation density

\section{Introduction}

Dislocation characterization is central to understanding plasticity. Dislocations are typically detected by observing the effect of localized lattice distortion fields via diffraction. For several decades, dislocations were characterized and quantified as discrete phenomena via transmission electron microscopy (TEM) [1]. While TEM techniques can detect distortion fields at length scales small enough to resolve individual dislocations, the leap from these localized measurements to the effect on macro, or even meso, response and related properties is difficult due to the large number of dislocations involved. Combined with the fact that sample preparation for TEM is both highly time consuming and destructive to the sample, denuding the sample of dislocations, a fully representative study of dislocations in bulk materials via TEM is problematic.

These difficulties have lead to efforts to understand dislocations at larger scales (typically at a scale where they can be modeled as continuous fields rather than discreet phenomena), techniques we refer to as continuum dislocation microscopy (CDM). CDM depends on the recovery of local lattice distortion gradients, which may then

\footnotetext{
${ }^{*}$ Corresponding author. Tel.: +1 8032709984

Email address: timmyruggs@gmail.com (T.J. Ruggles)
}

be related to the geometrically necessary dislocation content. With the advent of micro X-ray diffraction ( $\mu$ XRD) (e.g., [2]) and automated electron backscatter diffraction (EBSD) microscopy [3], information about the lattice orientation can be gleaned at relevant length scales. The advent of High Resolution EBSD (HREBSD) improved the resolution of this technique dramatically [4]. Using continuum dislocation mechanics developed by Nye and Kröner, this lattice information can be related to the dislocation content of the material [5]. These techniques were developed into the first continuum dislocation microscopy technology [6-11]. Calculated dislocation densities may be used to evaluate existing plasticity models [12] or applied directly to meso-scale calculations that incorporate GND evolution and effects $[13,14]$. This paper will examine EBSD CDM, a technique favored because of its superior spatial resolution as compared to $\mu \operatorname{XRD}[15,16]$.

Note that CDM only observes geometrically necessary dislocations (GNDs). GNDs are dislocations that are associated with long range distortion gradients in a material, relating to heterogeneous deformation [17]. Alternatively, statistically stored dislocations (SSDs) have no long range geometric effect because their distortion fields are canceled by other dislocations, and they are associated with homogeneous deformation. There is no physical difference between SSDs and GNDs; they are only distinguished by their distribution. This may be understood by 
imagining a large Burgers circuit. All dislocations within the circuit whose distortion effects are not canceled by other dislocations are GNDs; these dislocations contribute to distortion gradients above the length scale of the Burgers circuit. Dislocations within the circuit that collectively cancel each other's distortion fields (effectively dislocation dipoles) are SSDs. If the circuit contains a single dislocation, as is the case for TEM dislocation imaging, then the dislocation is a GND at that length scale. If a larger size of Burgers circuit is chosen, more dislocations within the circuit will match with dislocations of opposite sign, forming dislocation dipoles with no net geometric effect; these dislocations are now SSDs. Hence, the distinction between SSDs and GNDs is length scale dependent. For EBSD, the size of the implicit Burgers circuit is related to the step size. Thus, the calculated GND density will depend on the step size used to measure the lattice distortion. This and other effects of step size on GND calculations are problematic for researchers. Because all dislocations contribute to plasticity, not just GNDs, efforts have been made to determine the total dislocation content using continuum dislocation microscopy, GNDs and SSDs. Examining the material at length scales small enough to measure the geometric effect of individual dislocations [18] and examining the distribution of measured stress in the material [19] have been proposed as methods of determining total dislocation density.

CDM uses relationships developed by Nye and Kröner to relate derivatives of the lattice distortion to the dislocation content $[5,6,20,21]$. Typically local gradients are approximated numerically from a grid of discrete measurement points where the incident radiation (either Xrays or electron beam) interacts with the material. The spacing of the raster, which we denote as $L$, not only affects the numerical accuracy of the gradient determination, but it defines the implicit Burgers circuit. This paper investigates the effect of this grid length scale (or step size) on calculations of GND density via EBSD CDM. Researchers have already shown that calculated dislocation density is a function of the step size between neighboring points of the EBSD scan [22-24]. Measured lattice distortion gradients are highly sensitive to both real lattice fluctuations and noise of various kinds when taken over a range of step sizes. Thus, efforts have focused on selecting a single optimal step size, or a range of "safe" step sizes, that are large enough that noise effects do not dominate, and small enough to resolve important deformation features $[18,25]$. However, reporting GND density for a single step size may not always be appropriate; some physical information may be better obtained by analyzing calculated GND content at a number of different length scales.

At small step sizes, the calculated GND density has been shown to be roughly proportional to the inverse of the step size of the lattice distortion numerical derivatives [22, 24]. For materials with very low dislocation density like epitaxial silicon, this relationship measured dislocation density and step size appears to be inversely proportional due to an approximately constant algorithmic noise in the measured distortion between points in the raster independent of step size between the points of interest. Any actual dislocation density in epitaxial materials is well below the noise threshold of most EBSD dislocation microscopy techniques. However, inverse behavior or approximately inverse behavior is also reported for engineering materials expected to have much higher levels of dislocation density. The transition of GNDs to SSDs with increasing step size has been proposed to explain this inverse relationship when measured dislocation densities are well above the expected noise threshold [18]. For a fairly homogeneous, random distribution of dislocations with no net dislocation content, the cancellation of distortion effects as more dislocations are included in a growing Burgers circuit suggests a $1 / L$ dependence, as will be shown later in this work.

It is well documented that plastic deformation typically leads to heterogeneous distributions of dislocations at some length scale - either due to pile up at grain boundaries or to dislocation substructure (cells, cell blocks, geometrically necessary boundaries, etc. [26, 27]) or due to heterogeneous deformation [28]. At the length scales of the heterogeneity, strain gradients are present. These gradients are associated with net dislocation content which may be thought of as "true" GNDs. Determination of these true GNDs is relatively insensitive to the step size used to quantify them. As the step size increases, the effects of homogeneously distributed dislocations will become insignificant (due to the nature of the $1 / L$ relationship) compared to the effect of heterogeneously distributed dislocations ("true" GNDs). Hence, a graph of dislocation density versus step size shows a transition from the $1 / L$ relationship to an almost constant GND density at moderately large step sizes (the exact threshold will depend on the relative amount of homogeneously distributed and heterogeneously distributed dislocations). This behavior has not been explicitly reported in literature; data supporting this argument will be presented here. These non-canceling true GNDs have also been incorporated into a statistical model of SSD to GND transition.

Once the measurement step size is greater than the char- 
acteristic length scale of the heterogeneously distributed dislocations, the level of detected GND density decreases because the step size is too large to resolve the substructure of the material. Additionally, for extremely large grains or single crystals, crystal symmetry can limit the measurable lattice distortion. Many HREBSD algorithms have a simple misorientation cutoff to avoid error associated with comparing patterns between different grains or subgrains. All of these effects lead to a second decrease in calculated dislocation density at very large step sizes. This decrease in the GND-step size relationship may also be modeled statistically to determine an upper bound to step size. The purpose of this work is to:

- Model the relationship between calculated GND content, total dislocation content, "true" GND content, and implicit Burgers circuit size using basic statistics;

- Apply this model to the calculated GND density versus step size curve of a heterogeneously deformed single crystal tantalum sample;

- Demonstrate criteria for EBSD step size selection;

- Use this statistical model to relate the measured GND density at various step sizes to the total dislocation content of the material.

\section{Background}

The lattice distortion gradient, measurable via EBSD, may be used to calculate the geometrically necessary dislocation content of a given crystalline material using continuum relationships developed by Nye and Kröner. It is typical in EBSD dislocation microscopy to simplify Nye's relationship by assuming that all dislocation activity can be represented as superpositions of pure edge and screw dislocation types. For example, an FCC material has 12 unique slip systems, combinations of slip direction and slip plane. If we assume that dislocations associated with these slip systems can be either pure edge or pure screw, then that suggests 24 unique dislocation types. However, because screw dislocations have the same dislocation line vector regardless of their slip plane, each screw dislocation may belong to one of two slip systems, meaning that there are effectively only 18 unique dislocation types, 12 edges and 6 screws [29]. Making this simplification, we state Nye's relationship as [20]:

$$
\alpha=\sum_{t=1}^{N} \rho^{t} \mathbf{b}^{t} \otimes \mathbf{r}^{t}
$$

where $\alpha$ is known as the Nye tensor. This tensor provides a concise representation of net dislocation density and type on a continuum level. The other terms in Equation $1, \rho^{t}, \mathbf{b}^{t}$, and $\mathbf{r}^{t}$, represent the dislocation density, Burgers vector and dislocation line vector of each dislocation type (indicated by $t$ ), respectively. Kröner then showed that the Nye tensor is related to the elastic distortion of the material as follows [21]:

$$
\alpha=\nabla \times \beta
$$

where $\beta$ is the elastic lattice distortion tensor (measurable via HREBSD techniques). Equation 2 is referred to as the fundamental relation of continuum dislocation theory.

The Nye-Kröner equations can be used with any method of local lattice measurement, including $\mu$ XRD [15] and precession electron diffraction in a TEM [30]. In this paper, the distortion gradients required by CDM are extracted via EBSD. EBSD works by directing the electron beam of a scanning electron microscope in a finely spaced raster over the surface of a crystalline sample tilted at $70^{\circ}$ relative to the beam (to maximize the intensity of the backscattered electrons[31]). The resulting backscattered diffraction pattern is collected using a phosphor screen and charge coupled device (CCD) camera and stored electronically. The individual patterns are then indexed, usually with automated commercial software, recovering the crystal orientation at each point in the scan. Conventional EBSD software indexes individual patterns to find the local lattice orientation to within about $0.1^{\circ}$ via 2D Hough transforms for best case scenarios, with $0.5^{\circ}$ being a conservative estimate [32-35]. This information may be used to approximate a distortion gradient if strain components of the deformation tensor are neglected [6].

HREBSD not only improves the angular resolution of EBSD, it also allows strain gradients to be calculated alongside rotations, which lead to more accurate dislocation determination [4]. HREBSD accomplishes this by measuring shifts between regions of interest on related patterns via cross correlation techniques, and subsequently relating these shifts to the relative deformation between the lattices. This allows neighboring points to be directly compared to calculate lattice distortion gradients. It should be noted that HREBSD typically calculates relative distortion and not absolute distortion, making it highly suitable for calculating gradients, but the recovery of absolute distortion depends on having a strain free pattern of appropriate orientation for reference. Fortunately, CDM only requires distortion gradients, which are readily obtainable from measurements of relative distortion, and 
not absolute distortion. Some efforts have been made to use strain free simulated EBSD patterns to calculate absolute distortion (and therefore absolute strain)[36-38], but the greater accuracy and decreased geometric uncertainty afforded by comparing neighboring real patterns instead of using simulated reference patterns is preferred when derivatives are desired. Researchers report that HREBSD has a theoretical accuracy to within about 50 microstrain when calculating relative distortion between two patterns [4]. Other factors may degrade pattern quality and lead to further decreased accuracy. Properties of the material being examined, microscope settings, dislocation content, phase, polishing technique and EBSD image binning all have an effect on pattern quality [16, 24, 39-41].

The relative distortion between patterns collected from neighboring points on a scan may be used to approximate the gradient of the distortion via the forward difference derivative:

$$
\left.\frac{d \beta}{d x_{i}}\right]_{\vec{p}}=\frac{\beta^{\vec{p}, \vec{p}+\Delta \vec{x}_{i}}}{L}
$$

where $\vec{p}$ is a location on the surface of a scan, $\Delta \vec{x}_{i}$ is the vector between the first pattern and an adjacent pattern, $L$ is the spacing between raster points and the magnitude of $\Delta \vec{x}_{i}$, and $\beta^{\vec{p}, \vec{p}+\Delta \vec{x}_{i}}$ is the relative distortion between patterns at location $\vec{p}$ and $\vec{p}+\Delta \vec{x}_{i}$. Once the distortion gradient is obtained, it may be related to the GND density. Conventionally, the bulk GND content of the material may be obtained by calculating the available components of the Nye tensor from Eq. 2 (based upon measured values of $\beta$ ), and then extracting the densities relating to individual dislocation types, $\rho^{t}$, from Eq. 1 . This method is problematic for two reasons. First, even relatively uncomplicated FCC materials have 18 unique combinations of a Burgers vector and a dislocation line vector whereas the Nye tensor has only nine terms, meaning the problem is underconstrained. This problem is far worse with BCC materials, like the tantalum in this study, because of the large number of potential slip planes. Secondly, all nine terms of the Nye tensor are not typically available because lattice distortion derivatives can only be calculated in two dimensions parallel to the sample surface without serial sectioning [42], which is time consuming and destructive to the sample. This makes the problem even more underconstrained, adding to the ambiguity of the solution.

Because Eq. 1 is underconstrained, additional constraints, typically incorporated in some form of optimization approach, must be employed to find a reasonable estimate of dislocation density [6, 7, 43, 44]. The two most common methods involve minimizing the $L_{2}$ norm of the individual dislocation densities or the $L_{1}$ norm of the dislocation densities $[6,45]$. The $L_{2}$ method, while having no explicit physical meaning, can be calculated efficiently using the pseudo-inverse; the $L_{1}$ approach is based upon minimizing total dislocation line length, and therefore total slip [46]. This study is only concerned with the bulk dislocation density, so it is unnecessary to deal with the optimization problem. An estimate of the overall bulk dislocation density is available without trying to resolve the density onto individual dislocation types. The entry-wise one-norm of $\alpha$ (estimated from its measurable elements only) and kernel average misorientation have been used to find reasonable estimates of bulk dislocation density without having to minimize the $L_{1}$ norm of the dislocation density solution vector [7, 11, 42, 47]. This study uses the entry-wise one-norm of the Nye tensor to estimate the bulk dislocation density as described in a previous study by the authors [11]:

$$
\rho \approx \frac{3}{2 b} \sum_{i} \sum_{j}\left|\alpha_{i j}\right|
$$

where $\rho$ is an estimate of the total bulk dislocation density (the sum of the dislocation density on each slip system), $b$ is the Burgers vector, and the unknown terms of the Nye tensor are estimated by assuming all unknown distortion terms are 0 . The factor $3 / 2$ compensates for the missing lattice distortion derivatives.

\section{Methods and materials}

Based on the CDM approach, GND densities can be obtained for the chosen raster step-size, which is also the size of the implicit Burgers circuit. As discussed above, the calculated GND density is generally a function of the selected step-size. In order to examine the effect of stepsize on dislocation density over a large range of step sizes, large grained or single crystal samples are preferred. For this study, a tantalum single crystal prepared by collaborators at Columbia University was used. The sample was deformed with a tungsten carbide wedge indenter with a $90^{\circ}$ included angle, sectioned and polished so that approximate plane-strain conditions exist on the EBSD surface. The face of the sample is parallel to the (101) plane and the wedge indenter was pushed in the [101] direction with the leading edge parallel to the $[\overline{101}]$ direction.

The sample was polished with oil-based diamond suspensions starting from a particle size of $9 \mu \mathrm{m}$ and finishing at $1 \mu \mathrm{m}$. After being ultrasonically cleaned in ethanol, the sample was etched with a $50 \%$ sulfuric acid, 
$25 \%$ nitric acid, $25 \%$ hydrofluoric acid volumetric solution at $18-20^{\circ} \mathrm{C}$. A $10 \times 10 \mu \mathrm{m}$ area of the sample 150 $\mu \mathrm{m}$ from the indentation (see Figure 1 to see the location of the scanned area on the sample) with a step size of $20 \mathrm{~nm}$ was scanned in a FEI Helios NanoLab ${ }^{\text {TM }} 600 \mathrm{i}$ scanning electron microscope using TSL's OIM $^{\mathrm{TM}}$ software and a high speed Hikari ${ }^{\mathrm{TM}}$ camera to gather EBSD patterns. Standard EBSD geometry and an accelerating voltage of $25 \mathrm{keV}$ were used. The binning size of the pixels on the phosphor screen was $2 \times 2$. The sample was scanned over a small area near the indenter tip to ensure a high amount of heterogeneous deformation, associated with GNDs and strain gradients. Dislocation density was calculated at each point using Eq. 4. Because it is impractical to conduct a large number of scans at various step sizes, the scans were post processed multiple times, increasing the step size by skipping points in the raster as in $[18,24]$. A brief experiment was conducted comparing real variation in the step size versus artificial variation of the step size by skipping points, and it was found that skipping points was similar to real variation in the step size.

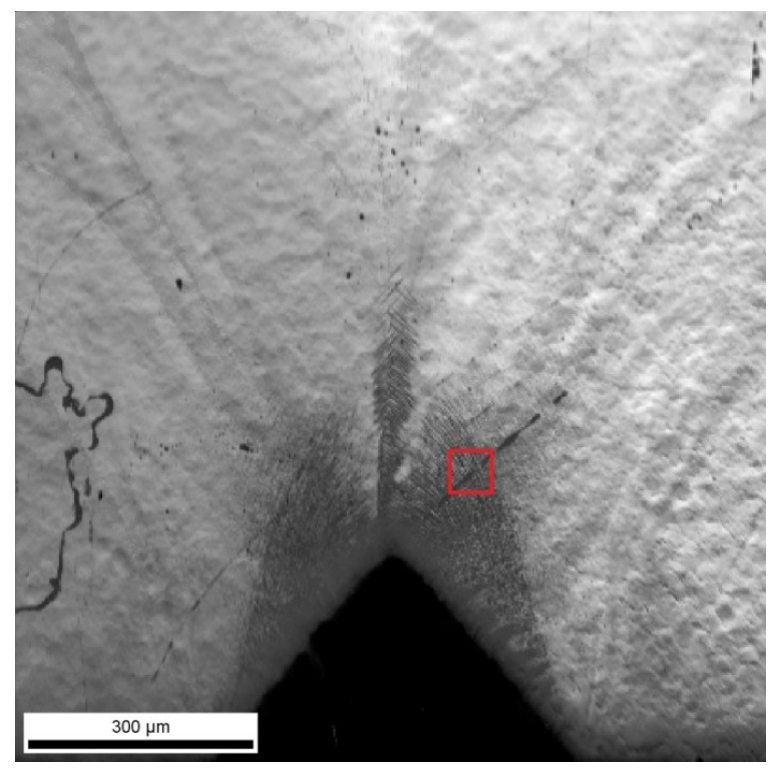

Figure 1: Electron micrograph of the indented Ta single crystal specimen used in this study. The general location of the scanned area is marked in red. The face of the specimen is parallel to the (101) plane and the indentation points in the [101] direction.

In addition to the tantalum single crystal described, this paper will also briefly investigate the dislocation density versus step size behavior for a number of other materials to show that the observations made in the paper are broadly applicable. Standard processes were used to prepare these materials and they were scanned with simi- lar parameters to the tantalum sample described. These samples include annealed magnesium, copper and iron, as well as epitaxial germanium.

\section{Results}

Dislocation density maps of the tantalum sample at selected step sizes are shown in Figure 2 and the average calculated GND density at each step size is plotted in Figure 3. In this paper, average will refer to the arithmetic mean. There is a complex relationship between the step size of an EBSD scan ( $L$ in Eq. 3$)$ and the calculated dislocation density ( $\rho$ in Eq. 4). If we think of this relationship as a curve for visualization purposes, we note three distinct regions of the curve across the length scales, only one of which (region A) has been represented in the literature so far. The three regions are labeled A, B and C on Figure 3. The mechanisms behind these three regions were discussed in the introduction, and they will be explained more thoroughly here.

At small step sizes, calculated GND density is roughly inversely proportional to step size. This relationship is attributed to some combination of algorithmic noise and/or the GND to SSD transition. For the tantalum sample, we expect the level of real dislocation density to be large compared to the algorithmic noise error, suggesting that the GND to SSD transition is the dominant mechanism behind region $\mathrm{A}$ in this sample. This region encompasses only a small portion of the entire curve because of the complex GND structure present. To show that the inverse step size relationship is near universal at small step sizes, EBSD scans from a number of different annealed materials were examined. Calculated GND versus step size curves for annealed magnesium, copper, and iron as well as epitaxial germanium at small step sizes are shown in Figure 4. Note that all of these curves are roughly parallel to the noise line, meaning they follow a $1 / L$ type relationship corresponding to region A from Figure 3. The $1 / L$ relationship even continues below the so called continuity limit, the point where less than one dislocation is contained on average in the implicit Burgers circuit. The copper sample examined shows an $L^{-0.8}$ relationship with dislocation density, which agrees with the work of Field, et al. [22], suggesting that the dislocations in copper are distributed differently.

There is a certain amount of noise in determining the relative distortion between two EBSD patterns using HREBSD which is associated with limited resolution of the phosphor screen, as mentioned in the background section. Low quality patterns will also contribute to noise in 


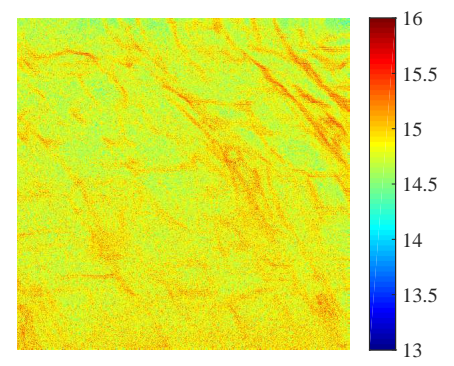

(a)

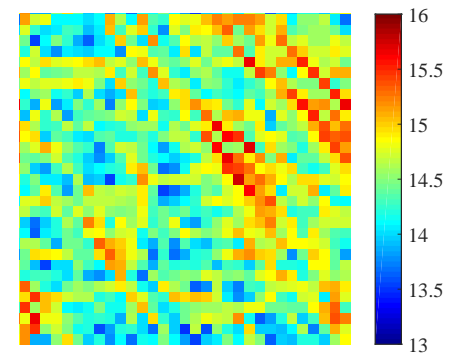

(c)

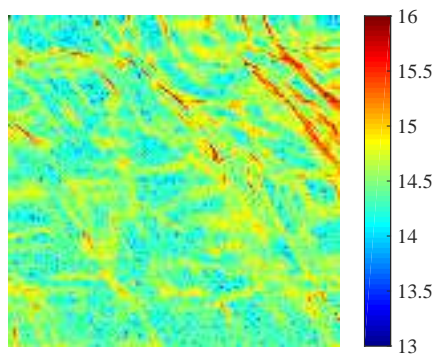

(b)

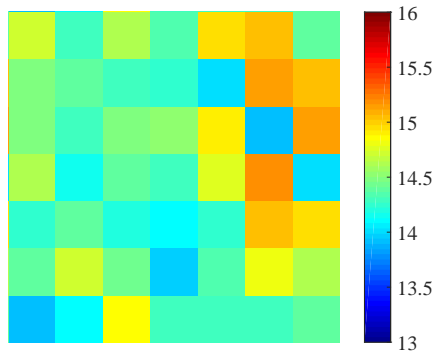

(d)

Figure 2: Dislocation density maps calculated at various step sizes for a $10 \times 10 \mu \mathrm{m}$ area beneath the indenter for a tantalum single crystal deformed by micro indentation. The dislocation density is presented in $\log$ units $\mathrm{of} \mathrm{m} / \mathrm{m}^{3}$. The dislocation density was calculated with a step size of (a) $20 \mathrm{~nm}$, (b) $80 \mathrm{~nm}$, (c) $320 \mathrm{~nm}$, and (d) $1280 \mathrm{~nm}$.

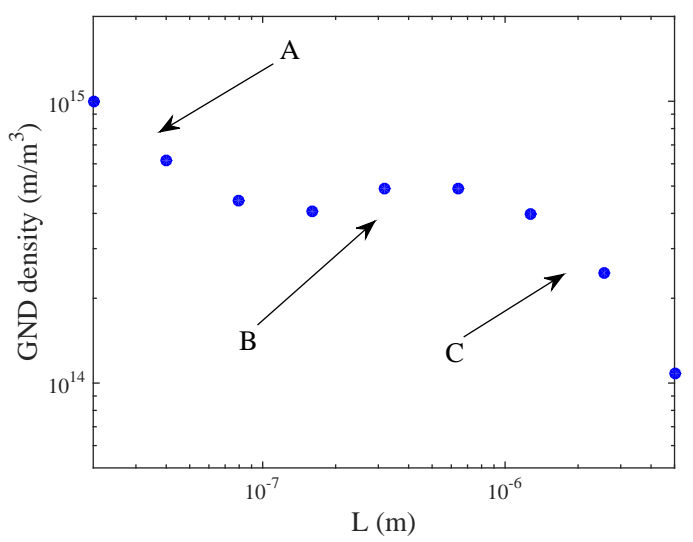

Figure 3: Average calculated GND density versus step size for a microindented single crystal of tantalum near the indenter tip. The (A) small step size $1 / L$ relationship, the (B) relatively constant GND density region at moderate step size, and the $(C)$ large step size decrease in dislocation density are all labelled.

the cross-correlations, but generally it is assumed for high quality scans that phosphor screen resolution error will be more significant. Wilkinson provides a rough estimate of the error in the distortion calculation due to phosphor screen resolution at around 50 microstrain [4] (Compare this to the worst case $0.5^{\circ}$ accuracy of conventional EBSD [32-35], which corresponds to almost 9000 microstrain).

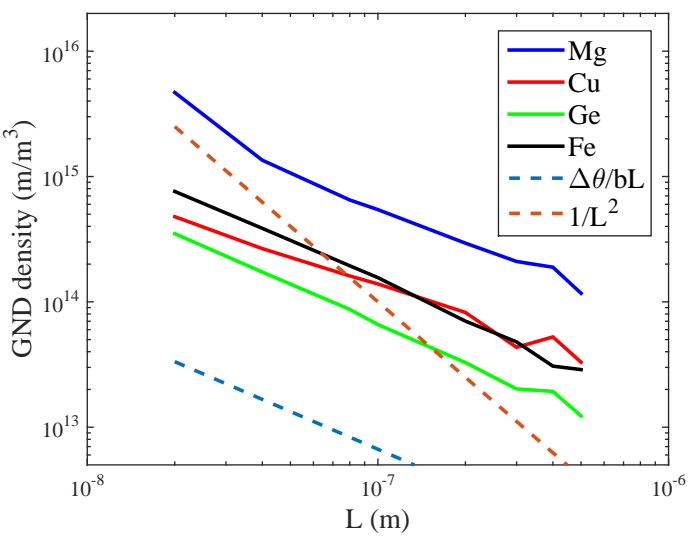

Figure 4: Calculated GND curves for annealed magnesium, copper, and iron as well as epitaxial germanium at small step sizes. For comparison, the postulated resolution limit due to the inherent noise in HREBSD $(\Delta \theta / b L)$ and the continuity limit $\left(1 / L^{2}\right)$, the point where less than one dislocation is captured in the implicit Burgers circuit, are also plotted.

When Jiang investigated this lower bound using an epitaxially grown $\mathrm{Si}$ sample (assumed to be nearly dislocation free), he detected errors about a factor of 2 higher [24]. Error in the calculation of the numerical derivative of lattice distortion could lead to the $1 / L$ relationship frequently observed (see Eq. 3). However, Jiang points out that this 
level of noise is still orders of magnitude lower than typically calculated values of dislocation density, suggesting GND to SSD transition is the dominant effect on the calculated GND density/step size relationship in deformed samples. For the annealed materials and epitaxial germanium in Figure 4, deconvolving these effects is more problematic, and not attempted here.

At larger, "moderate" step sizes, the $1 / L$ relationship gives way to a region where the calculated GND density is roughly constant, region B in Figure 3. We postulated in the introduction that this constant region is the result of "true" GNDs that accommodate strain gradients on the scale of the substructure of the material, as in [18]. What constitutes a "small" or "moderate" step size depends on the nature and distribution of the dislocation content of the material, and will be further explained in the next section. For heterogeneously deformed samples like the tantalum in this study, there are more "true" GNDs and therefore a more prominent $B$ region than in the annealed samples. This is why the $\mathrm{B}$ region begins in the dislocation density curve for tantalum at approximately 100 to $200 \mathrm{~nm}$, while no constant region is detected in the annealed materials. Finally, at "large" step sizes (again, the transition between these various regions depends on the nature and quantity of deformation of the sample), the constant region gives way to yet another $1 / L$ type region. This second decreasing region is only observed at very large step sizes, often larger than the grain size of most engineering materials, and thus is not frequently observed. This region of the curve is visible in the GND curve of the micro-indented single crystal of tantalum seen in Figure 3.

\section{Analysis}

In this section, we will model the various regions of the calculated GND versus step size curve statistically. The GND to SSD transition seems to be the most likely explanation for the observed variation in the $\mathrm{A}$ and $\mathrm{B}$ regions of the calculated GND density curve for this sample. Modeling the curve as a function of the GND and SSD content of the material will allow the extraction of SSD information via EBSD, as well as provide criteria for step size selection. Adams and Kacher attempted to model this transition with a simple 2D Monte Carlo simulation [18], which modeled dislocations of a single type passing through a plane as points with either a positive or negative character. Dislocations of each sign are randomly distributed with more dislocations of positive than negative sign being simulated so that there are net, geometrically necessary dislocations at every length scale. The net disloca- tion density is calculated inside a number of boxes with varying area by subtracting the included negative dislocations from the included positive dislocations, taking the absolute value, and dividing by the area of the box. This side length of the box is analogous to the step size of the lattice distortion numerical derivative for a real scan. The average calculated GND density from a large number of boxes can then be plotted versus the step size. Here, we have duplicated the 2D Monte Carlo simulation of Adams and Kacher [18], as shown in Figure 5. The simulated total dislocation density is $3.125 \times 10^{13} \mathrm{~m} / \mathrm{m}^{3}$ (calculated GND density converges to this value at small step sizes in the graph) and the density of unpaired "true" GNDs is $6.25 \times 10^{12} \mathrm{~m} / \mathrm{m}^{3}$ (calculated GND density converges to this value at large step sizes in the graph).

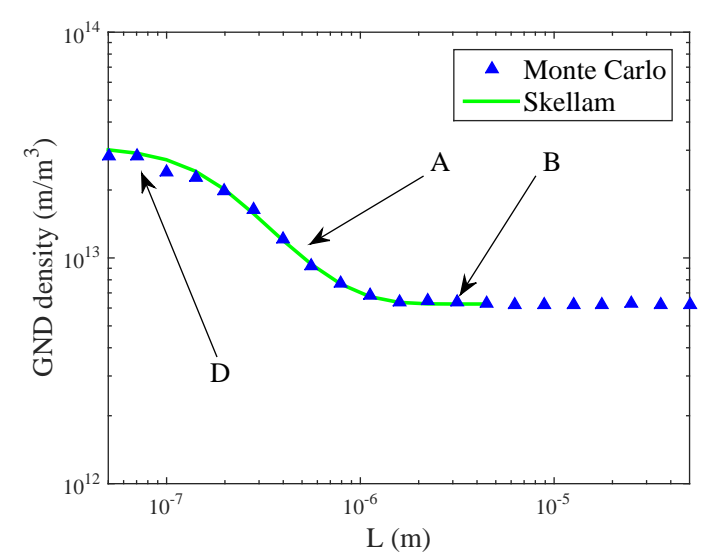

Figure 5: Calculated GND density versus step size for the Monte Carlo simulation as well as the Skellam distribution model. The simulated total dislocation density is $3.125 \times 10^{13} \mathrm{~m} / \mathrm{m}^{3}$ and the density of unpaired "true" GNDs is $6.25 \times 10^{12} \mathrm{~m} / \mathrm{m}^{3}$.

The relationship between detected dislocation density and step size may also be calculated analytically. For a randomly distributed field of positive dislocations with density $\rho^{+}$, the average number of dislocations in an $L \times L$ region will be represented by a Poisson distribution, which expresses the probability of a number of random events occurring in a region of space. The number of negative dislocations will also be characterized by a Poisson distribution. The expected value (the expected value of a Poisson distribution is also the variance) for each of these distributions is:

$$
\begin{aligned}
& \lambda^{+}=\rho^{+} L^{2} \\
& \lambda^{-}=\rho^{-} L^{2}
\end{aligned}
$$

In order to understand the net dislocation density, we will need to take the difference between the number of positive and the number of negative dislocations inside the 
square. The difference between two variables each having a Poisson distribution is a Skellam distribution. The Skellam distribution for the number of net dislocations is as follows:

$$
P(k, L)=\exp \left(\rho^{+} L^{2}+\rho^{-} L^{2}\right)\left(\frac{\rho^{+}}{\rho^{-}}\right)^{k / 2} I_{|k|}\left(2 \sqrt{\rho^{+} \rho^{-} L^{4}}\right)
$$

In this equation, $P(k, L)$ is the probability of $k$ net dislocations lying within an $L \times L$ square on the plane and $I$ is the modified Bessel function of the first kind. Finally, the average dislocation density may be calculated by taking the average of the absolute value of the Skellam distribution and scaling by the box area as follows:

$$
\bar{\rho}_{m}(L)=\frac{\sum_{-\infty}^{\infty}|k P(k, L)|}{L^{2}}
$$

Using Eq. 8 to calculate the expected detectable GND density agrees well with the 2D Monte Carlo simulation (see Figure 5). At large step sizes, the numerator of Eq. 8 becomes too large to calculate using a double precision variable, and the result is not defined, which is why the Skellam model is not calculated for the entire range of the Monte Carlo simulation. The Skellam model exhibits an A and B type region for GND density versus step size (see Figure 3). However, the Skellam model and the Monte Carlo simulation also have a constant region at very small step sizes (labeled D on Figure 5) of the same magnitude as the total simulated dislocation density. The Skellam model fails to accurately model the second decrease (region $\mathrm{C}$ in Figure 3). Calculating points on the curve is computationally expensive and numerically unstable. The Skellam model is valuable because it is intuitive, but it is not practical to apply to real data because of these reasons. We will instead adapt this model to more accurately reflect real HREBSD CDM, which will lead to greater verisimilitude as well as improved computational efficiency.

It should be noted that EBSD dislocation microscopy does not work by counting discrete dislocations, rather by measuring continuous distortion derivatives. As such, the discreet nature of the simulation and the Poisson and Skellam distributions is perhaps not the best means of modeling the GND step size relationship at very small step sizes in real materials. Discreet counting is responsible for the flat region at small step sizes in the Monte Carlo simulations and the Skellam model seen in Figure 5. Consequently, we have modified our model by replacing the discreet Skellam distribution with the continuous Gaussian normal distribution closest to the Skellam distribution. We model the number of detectable dislocations of a certain sign inside of an $L \times L$ square as a normal distribution with mean and variance both equal to $\lambda$ (which is approximately equivalent to the Poisson distribution for large $\lambda$ ). The difference between the number of positive and negative dislocations in the $L \times L$ square will also be a normally distributed variable with a mean of $\rho_{n} L^{2}$ and a variance of $\rho_{t} L^{2}$, where $\rho_{n}=\rho^{+}-\rho^{-}$and $\rho_{t}=\rho^{+}+\rho^{-}$. The variable $\rho_{n}$ may be thought of as the net dislocation density or "true" GND density discussed in the introduction and $\rho_{t}$ is the total dislocation density. Making these substitutions, the average calculable dislocation density may be determined as:

$$
\begin{aligned}
\bar{\rho}_{m}(L) & =\frac{\int_{-\infty}^{\infty}\left|x f\left(x, \rho_{t} L^{2}, \rho_{n} L^{2}\right)\right| d x}{L^{2}} \\
& =\sqrt{\frac{2}{\pi}} \exp \left(\frac{-\rho_{n}^{2} L^{2}}{2 \rho_{t}}\right) \frac{\sqrt{\rho_{t}}}{L}+\rho_{n} \operatorname{erf}\left(\frac{\rho_{n} L}{\sqrt{2 \rho_{t}}}\right)
\end{aligned}
$$

where $f\left(x, \sigma^{2}, \mu\right)$ is the Gaussian normal distribution probability density function for a variable, $x$, with a mean $\mu$ and a variance $\sigma^{2}$, and $\operatorname{erf}(z)$ is the error function. Our simplified model has led to the conclusion that the total dislocation density is proportional to the variance of local dislocation measurements. This agrees with the observation of other researchers that the variance of measured lattice strains is proportional to dislocation density [19, 48]. Equation 9 has the advantage of being much more efficient to calculate than the Skellam model (Eq. 8) due to the integrable nature of the Gaussian distribution. This function is plotted in Figure 6 along with the Skellam model from Figure 5. Note that at large step sizes, this formulation (Eq. 9) is equivalent to the Skellam distribution fit (Eq. 8) because a Skellam distribution approaches a Gaussian distribution for large expected values, but at smaller step sizes where the Skellam-based curve and the simulation converge to total dislocation density, Eq. 9 remains a $1 / L$ type relationship. The behavior of the Gaussian model better fits the behavior of real samples, as shown in Section 4. Even though the Gaussian model does not converge at small step sizes to the total dislocation density, this value still determines the magnitude of the A region.

Our improved model, which employs a Gaussian normal distribution instead of a Skellam distribution, more accurately models real GND density versus step size curves like those seen in the previous section while also being more numerically efficient. However, it still does not model the second decreasing region of these curves (i.e. region $\mathrm{C}$ in Figure 3). This region of the calculated GND density vs. step size relationship is typically only observed at step sizes larger than the grain size of 


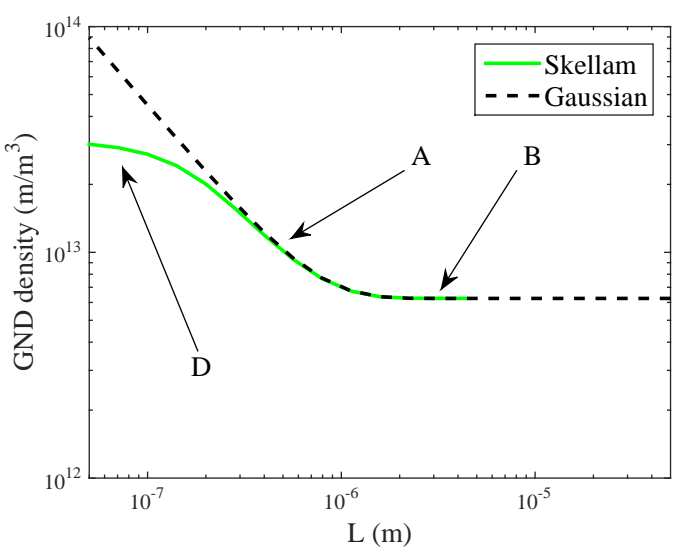

Figure 6: This figure shows the Gaussian model of a GND versus step size curve as well as the Skellam model. The dislocation density parameters are the same as in Figure 5.

most engineering materials, so this behavior is usually only seen in large-grained or single crystal samples. We propose two explanations for the $\mathrm{C}$ region of the curve. The first is the fact that distortion gradients associated with misorientations of greater than a certain threshold (in the case of this study $55^{\circ}$ ) are disregarded. Considering misorientations beyond $5^{\circ}$ exceeds the limits of infinitesimally small distortion (an assumption made in conventional HREBSD), and are too great to reliably recover a numerical derivative via forward difference. In other cases, the decrease in dislocation density may be due to dislocation substructure within the grain. If we imagine the dislocation substructure to be some pseudo periodic waveform, the numerical derivative will begin to decrease when the step size exceeds the characteristic length scale of the structure.

These two effects may be modeled jointly by simply adding a limit to the maximum measurable distortion between patterns. This effect may be simulated in the model simply by modifying the bounds of integration in Eq. 9, as follows:

$$
\begin{gathered}
\bar{\rho}_{m}(L)=\frac{\int_{-q L}^{q L}\left|x f\left(x, \rho_{t} L^{2}, \rho_{n} L^{2}\right)\right| d x}{L^{2}} \\
=\frac{\sqrt{\rho_{t}}}{L \sqrt{2 \pi}} \frac{2 \exp \left(\frac{-\rho_{n}^{2} L^{2}}{2 \rho_{t}}\right)-\exp \left(\frac{-\left(q+\rho_{n} L\right)^{2}}{2 \rho_{t}}\right)-\exp \left(\frac{-\left(q-\rho_{n} L\right)^{2}}{2 \rho_{t}}\right)}{\frac{1}{2} \operatorname{erf}\left(\frac{q-\rho_{n} L}{\sqrt{2 \rho_{t}}}\right)+\frac{1}{2} \operatorname{erf}\left(\frac{q+\rho_{n} L}{\sqrt{2 \rho_{t}}}\right)} \\
+\rho_{n} \frac{\operatorname{erf}\left(\frac{\rho_{n} L}{\sqrt{2 \rho_{t}}}\right)-\frac{1}{2} \operatorname{erf}\left(\frac{q+\rho_{n} L}{\sqrt{2 \rho_{t}}}\right)-\frac{1}{2} \operatorname{erf}\left(\frac{q-\rho_{n} L}{\sqrt{2 \rho_{t}}}\right)}{\frac{1}{2} \operatorname{erf}\left(\frac{q-\rho_{n} L}{\sqrt{2 \rho_{t}}}\right)+\frac{1}{2} \operatorname{erf}\left(\frac{q+\rho_{n} L}{\sqrt{2 \rho_{t}}}\right)}
\end{gathered}
$$

where $q$ is the maximum amount of detectable lattice distortion between patterns divided by the magnitude of
Burgers vector. The resulting equation is obviously quite unwieldy and has computational overflow issues at large step sizes (precisely where it differentiates from Eq. 9) because the exponentials in the numerator of the first term would become too large for double precision variables before being divided. Making the following substitution will allow us to simplify this equation:

$$
\operatorname{erf}(z)=1-\exp \left(-z^{2}\right) \operatorname{erfcx}(z)
$$

where $\operatorname{erfcx}(z)$ is the scaled complimentary error function. Making this substitution and by assuming that $L$ is large, Eq. 10 may be simplified to Eq. 12, which is stable at large step sizes:

$$
\begin{aligned}
\bar{\rho}_{m}(L)=\frac{\int_{-q L}^{q L}\left|x f\left(x, \rho_{t} L^{2}, \rho_{n} L^{2}\right)\right| d x}{L^{2}} \\
\approx \rho_{n}-\sqrt{\frac{2}{\pi}} \frac{\sqrt{\rho_{t}}}{\operatorname{erfcx}\left(\frac{\rho_{n} L-q}{\sqrt{2 \rho_{t}}}\right) L}
\end{aligned}
$$

Equation 12 is valid only for the $\mathrm{B}$ and $\mathrm{C}$ regions of the calculated GND density curve, but it has no overflow problems like Eq. 10. Due to the sigmoid nature of these curves, Eq. 12 may actually be combined with Eq. 9 despite the fact that both of these equations may be thought of as simplifications of Eq. 10 with antagonistic assumptions (Eq. 9 makes the assumption that $q$ is large and Eq. 12 assumes $L$ is large). The resulting equation is identical to Eq. 10 to within numerical error, but has no computational overflow problems:

$$
\begin{array}{r}
\bar{\rho}_{m}(L) \approx \\
\frac{\sqrt{2 \rho_{t}}}{L \sqrt{\pi}}\left(\exp \left(\frac{-\rho_{n}^{2} L^{2}}{2 \rho_{t}}\right)-\frac{1}{\operatorname{erfcx}\left(\frac{\rho_{n} L-q}{\sqrt{2 \rho_{t}}}\right)}\right) \\
+\rho_{n} \operatorname{erf}\left(\frac{\rho_{n} L}{\sqrt{2 \rho_{t}}}\right)
\end{array}
$$

This modified form of the Gaussian method (Eq. 13) is compared to Eq. 9 in Figure 7. Since our Monte Carlo simulation does not restrict the detectable number of dislocations and because there is no substructure in the simulated dislocation fields, the limit parameter is completely arbitrary to show the potential of the equation. Because it is difficult to deconvolve the various factors that contribute to this limit and because this region of the curve contains little useful dislocation information, we will use $q$ only as a fitting parameter on real samples.

This model may now be fit to real data using only three 


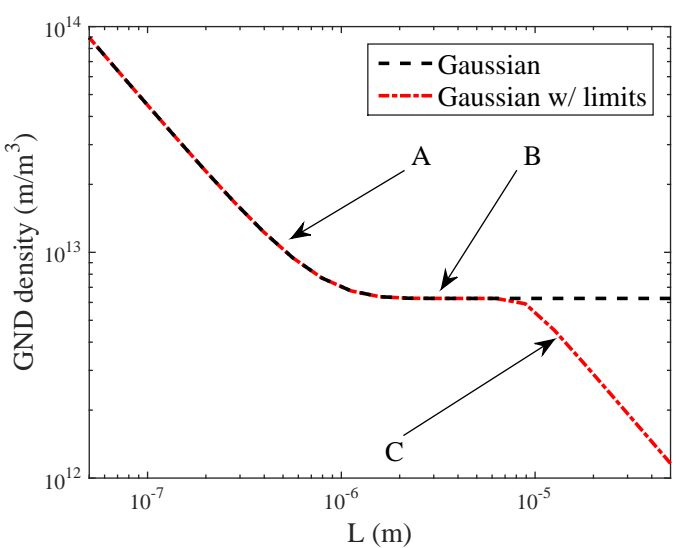

Figure 7: The Gaussian model of a GND density versus step size curve is compared with the Gaussian model with limits of integration applied. Note that the two models are indistinguishable until larger step sizes where the model with limits applied decreases, as seen in real data (see Figure 3).

parameters, total dislocation density, "true" GND density, and $q$. Thus, generating a GND density versus step size curve and fitting this model to it allows us to estimate not only GND density associated with strain gradients, but also the total dislocation density. We fit our Gaussian model to the dislocation densities for the indented tantalum sample shown in Figure 3. The fit was performed by optimizing the three parameters to minimize the sum of the squared error in log space (to fit all regions of the data). The parameters of the curve show that the average total dislocation density over the area of the scan is $6.02 \times 10^{14} \mathrm{~m} / \mathrm{m}^{3}$ and the density of heterogeneously distributed dislocations ("true" GNDs) is $4.36 \times 10^{14} \mathrm{~m} / \mathrm{m}^{3}$. The fit is shown in Figure 8. The three parameters reasonably fit the $\mathrm{A}, \mathrm{B}$ and $\mathrm{C}$ regions of the data. However, there

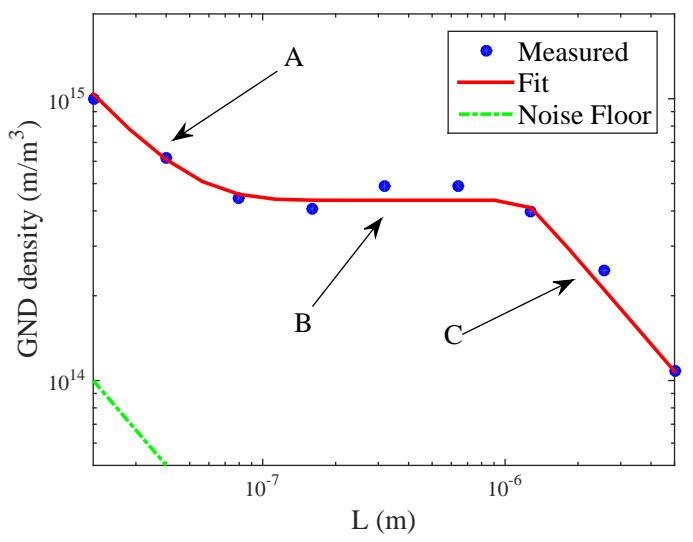

Figure 8: GND density versus step size for an indented tantalum sample (see Figure 3) fit with the Gaussian model with limits. The parameters used to fit the curve are $\rho_{n}=4.36 \times 10^{14} \mathrm{~m} / \mathrm{m}^{3}, \rho_{t}=$ $6.02 \times 10^{14} \mathrm{~m} / \mathrm{m}^{3}$, and $q=5.39 \times 10^{8} \mathrm{~m}^{-1}$ are some features in the data that the model does not account for. The B region, for example, is not perfectly flat, actually increasing for a small range of step size. This discrepancy is most likely related to the fact that there is not simply a smooth gradient across the sample. A more complex model would incorporate dislocation substructure more explicitly. It should also be noted that the statistical model is unable to distinguish noise from total dislocation content, and relies on the assumption that the noise is insignificant. In situations where the noise level is expected to be higher relative to the total dislocation density the fit should be applied with care. This can happen for a number of reasons, for example low dislocation density in the sample, difficult to prepare material like magnesium, and microscope configurations or camera binning which are selected for speed, rather than pattern quality. The exact relationship between pattern quality and noise in the dislocation measurements can be difficult to predict and model.

Current practice is to assume that the noise level in the dislocation density measurements is determined as follows [18]:

$$
\rho_{\text {noise }}=\frac{\theta}{b L}
$$

where $\theta$ is the angular resolution of HREBSD, $b$ is Burgers vector, $L$ is step size and $\rho_{\text {noise }}$ works as a threshold below which measurements are considered unreliable, the noise floor. The theoretical angular resolution of HREBSD is around $7 \times 10^{-5}$ rad for standard resolution CCD cameras [4]. The patterns used in this study were taken with a $464 \times 464$ camera but binned $2 \times 2$, meaning the effective resolution was $232 \times 232$. Jiang, et al. conducted a study into the effects of camera binning and found that for this resolution the estimated angular resolution is around $6 \times 10^{-4} \mathrm{rad}$ [24]. The noise floor assuming this angular resolution is plotted in Figure 8 using Equation 14. Note that this noise estimation is never greater than $10 \%$ of the measured dislocation density, suggesting that that neglecting noise was justified in our model. However, estimations of angular resolution are difficult to verify and are typically overly optimistic, even in studies of materials with high pattern quality like epitaxial silicon. The real angular resolution of an EBSD scan is difficult to determine and would require consideration of the pattern quality; the values stated here are only an estimate. Additionally, the relationship between dislocation density measurements and angular resolution, Equation 14, is only an approximation. More work is required to reliably quantify noise effects in order to more confidently calculate total dislocation density. 


\section{Conclusions}

The variation in EBSD measurements of GND content due to the step size of the numerical derivative of the lattice distortion has been reported multiple times in the literature [18, 22-24]. A number of phenomena have been suggested to explain this complex relationship, including noise in the calculation of the lattice distortion due to the resolution limit of the phosphor screen and periodic dislocation structure of a given length scale. This study has found that most variation of the calculated GND density may be statistically modeled as the shift of GNDs to SSDs for a randomly distributed field of dislocations. The relationship between heterogeneous and homogeneous dislocation content and the calculated dislocation density is encapsulated in Eq. 9. For very large step sizes and/or higher levels of heterogeneously distributed dislocations, other effects must be taken into account via Eq. 13.

We applied the model by fitting it to calculated GND density versus step size data from an indented tantalum sample, which suggested that the area scanned had a total dislocation density of $6.02 \times 10^{14} \mathrm{~m} / \mathrm{m}^{3}$ and a "true" GND density of $4.36 \times 10^{14} \mathrm{~m} / \mathrm{m}^{3}$. The fit of this curve suggested an unusually high ratio of "true" GNDs associated with heterogeneous deformation compared with typical homogeneously distributed dislocations. Most available data in the literature concerns specimens deformed in simple tension, where the deformation is more homogeneous and they do not detect a B region. The prominent $\mathrm{B}$ region in our heterogeneously deformed sample is consistent with the claim that the B region of GND density curves is associated with "true" geometrically necessary dislocations.

The model allows us to propose robust criteria for step size selection in EBSD dislocation microscopy studies. Once a GND density versus step size curve is generated by skipping raster points, the $\mathrm{B}$ region, where dislocation density is relatively insensitive to small changes in step size, may be identified. This constant region represents a range of potential step sizes that allow the accurate recovery of the "true" GND density, dislocation density associated with deformation heterogeneity at the length scale of the grain. Perhaps more significantly, the statistical model of GND to SSD transition provides a new estimate of the total bulk dislocation density of a material. Because all dislocations contribute to the plastic behavior of materials, this represents a significant advance in EBSD dislocation microscopy technology.

The model has significant room for refinement. Because it fails to include the effects of noise and substruc- ture, it is not suitable for all situations. The various factors that contribute to the limit parameter, $q$, have also not been well defined. Error due to geometrical uncertainty has also been neglected. The hope is that the statistical framework presented here is flexible enough to incorporate these effects as the limits of EBSD based dislocation microscopy become more fully understood.

\section{Acknowledgments}

Research was supported by U.S. Department of Energy (DOE), Office of Science, Basic Energy Sciences (BES), under Award \#DE-SC0012587 (computational studies and theory development), and by the National Science Foundation (NSF), under award \#CMMI 1404771 (experimental data gathering activities). The authors would also like to thank Jeff Kysar and his research group at Columbia for the preparation of the tantalum sample and Stuart Wright of EDAX/TSL for many helpful discussions.

\section{References}

[1] P. B. Hirsch, A. Howie, R. Nicholson, D. Pashley, M. J. Whelan, Electron microscopy of thin crystals, Butterworth, Washington, D.C., 1966.

[2] R. Tissot, Microdiffraction applications utilizing a twodimensional proportional detector, Power Diffraction 18 (2003) 86-90.

[3] B. L. Adams, S. I. Wright, K. Kunze, Orientation imaging: the emergence of a new microscopy, Metallurgical Transactions A 24 (4) (1993) 819-831.

[4] A. J. Wilkinson, G. Meaden, D. J. Dingley, High resolution mapping of strains and rotations using electron back scatter diffraction, Materials Science and Technology 22 (11) (2006) 1-11.

[5] B. L. Adams, Orientation imaging microscopy: Emerging and future applications, Ultramicroscopy 67 (1-4) (1997) 11-17.

[6] S. Sun, B. Adams, W. King, Observations of lattice curvature near the interface of a deformed aluminium bicrystal, Philosophical Magazine A: Physics of Condensed Matter, Structure, Defects and Mechanical Properties 80 (1) (2000) 9-25.

[7] B. El-Dasher, B. Adams, A. Rollett, Viewpoint: Experimental recovery of geometrically necessary dislocation density in polycrystals, Scripta Materialia 48 (2) (2003) 141-145.

[8] D. Field, P. Trivedi, S. Wright, M. Kumar, Analysis of local orientation gradients in deformed single crystals, Ultramicroscopy 103 (2005) 33-39.

[9] C. J. Gardner, B. L. Adams, J. Basinger, D. T. Fullwood, EBSDbased continuum dislocation microscopy, International Journal of Plasticity 26 (2010) 1234-1247.

[10] A. Wilkinson, E. Clarke, T. Britton, P. Littlewood, P. Karamched, High-resolution electron backscatter diffraction: an emerging tool for studying local deformation, The Journal of Strain Analysis for Engineering Design 45 (2010) 365-376.

[11] T. J. Ruggles, D. T. Fullwood, Estimations of bulk geometrically necessary dislocation density using high resolution EBSD, Ultramicroscopy 133 (2013) 8-15. 
[12] J. Jiang, T. B. Britton, A. J. Wilkinson, The orientation and strain dependence of dislocation structure evolution in monotonically deformed polycrystalline copper, International Journal of Plasticity 69 (2015) 102-117.

[13] H. Lim, S. Subedi, D. Fullwood, B. Adams, R. Wagoner, A practical meso-scale polycrystal model to predict dislocation densities and the Hall-Petch effect, Materials Transactions 55 (1) (2014) 35-38.

[14] T. Zhang, D. M. Collins, F. P. Dunne, B. A. Shollock, Crystal plasticity and high-resolution electron backscatter diffraction analysis of full-field polycrystal $\mathrm{Ni}$ superalloy strains and rotations under thermal loading, Acta Materialia 80 (0) (2014) 25 38.

[15] P. Lynch, D. Tomus, C. Bettles, M. Gibson, A. Stevenson, A comparative EBSD and micro-XRD study of the intergranular grain structure in CP-Ti, Nuclear Instruments and Methods in Physics Research A 619 (2010) 298-301.

[16] A. Schwartz, M. Kumar, B. Adams, D. Field, Electron backscatter diffraction in materials science, 2nd Edition, Springer, New York, 2009.

[17] M. F. Ashby, The deformation of plastically non-homogeneous materials, Philosophy Magazine 21 (1970) 399-424.

[18] B. Adams, J. Kacher, EBSD-based microscopy: Resolution of dislocation density, Computers, Materials and Continua 14 (3) (2010) 185-196.

[19] A. J. Wilkinson, E. Tarleton, A. Vilalta-Clemente, J. Jiang, T. B. Britton, D. M. Collins, Measurement of probability distributions for internal stresses in dislocated crystals, Applied Physics Letters 105 (18) (2014) -. doi:http://dx.doi.org/10.1063/1.4901219.

[20] J. Nye, Some geometrical relations in dislocated crystals, Acta Metallurgica 1 (1953) 153-162.

[21] E. Kröner, Continuum theory of dislocations and self-stresses, Ergebnisse der Angewandten Mathematik 5 (1958) 1327-1347.

[22] D. Field, C. Merriman, N. Allain-Bonasso, F. Wagner, Quantification of dislocation structure heterogeneity in deformed polycrystals by EBSD, Modelling and Simulation in Materials Science and Engineering 20 (2012) 12pp.

[23] D. Field, C. Merriman, J. Smith, Excess dislocation density measurement dependence on EBSD step size, Microsc Microanal 13 (2007) 920-921.

[24] J. Jiang, T. Britton, A. Wilkinson, Measurement of geometrically necessary dislocation density with high resolution electron backscatter diffraction: Effects of detector binning and step size, Ultramicroscopy 125 (2013) 1-9.

[25] J. Kysar, Y. Saito, M. Oztop, D. Lee, W. Huh, Experimental lower bounds on geometrically necessary dislocation density, International Journal of Plasticity 26 (2010) 1097-1123.

[26] I. Gutierrez-Urrutia, D. Raabe, Multistage strain hardening through dislocation substructure and twinning in a high strength and ductile weight-reduced Fe-Mn-Al-C steel, Acta Materialia 60 (16) (2012) 5791-5802.

[27] D. Hughes, N. Hansen, D. Bammann, Geometrically necessary boundaries, incidental dislocation boundaries and geometrically necessary dislocations, Scripta Materialia 48 (2) (2003) 147153.

[28] J. W. Kysar, Y. Gan, T. Morse, X. Chen, M. Jones, High strain gradient plasticity associated with wedge indentation into facecentered cubic single crystals: Geometrically necessary dislocation densities, Journal of the Mechanics and Physics of Solids 55 (2007) 1554-1573.

[29] L. Evers, W. Brekelmans, M. Geers, Scale dependent crystal plasticity framework with dislocation density and grain boundary effects, International Journal of Solids and Structures 41 (2004) 5209-5230.

[30] A. Leff, C. Weinberger, M. Taheri, Estimation of dislocation density from precession electron diffraction data using the Nye tensor, Ultramicroscopy 153 (0) (2015) $9-21$.

[31] M. N. Alam, M. Blackman, D. W. Pashley, High-angle kikuchi patterns, Proceedings of the Royal Society A 221 (1954) 224242.

[32] F. Ram, S. Zaefferer, D. Raabe, Kikuchi bandlet method for the accurate deconvolution and localization of Kikuchi bands in Kikuchi diffraction patterns, Journal of Applied Crystallography 47 (2014) 264-275.

[33] S. Wright, M. Nowell, High-speed EBSD, Advanced Materials and Processes 166 (2) (2008) 29-31.

[34] S. Wright, M. Nowell, J. Basinger, Precision of EBSD based orientaion measurements, Microscopy and Microanalysis 17 (2011) 406-407.

[35] S. I. Wright, M. M. Nowell, R. de Kloe, L. Chan, Orientation precision of electron backscatter diffraction measurements near grain boundaries, Microscopy and Microanalysis 20 (2014) 852863.

[36] J. Kacher, C. Landon, B. L. Adams, D. Fullwood, Braggs law diffraction simulations for electron backscatter diffraction analysis, Ultramicroscopy 109 (9) (2009) 1148-1156.

[37] T. Britton, C. Maurice, R. Fortunier, J. Driver, A. Day, G. Meaden, D. Dingley, K. Mingard, A. Wilkinson, Factors affecting the accuracy of high resolution electron backscatter diffraction when using simulated patterns, Ultramicroscopy 110 (2010) 1443-1453.

[38] D. Fullwood, M. Vaudin, C. Daniels, T. Ruggles, S. I. Wright, Validation of kinematically simulated pattern HR-EBSD for measuring absolute strains and lattice tetragonality, Materials Characterization 107 (2015) 270 - 277.

[39] M. Nowell, R. Witt, B. True, EBSD sample preparation: Techniques, tips and tricks, Microscopy and Microanalysis 11 (Suppl 2).

[40] L. Ryde, Application of EBSD to analysis of microstructures in commercial steels, Materials Science and Technology 22 (11) (2006) 1297-1306.

[41] R. Yoda, T. Yokomaku, N. Tsuji, Plastic deformation and creep damage evaluations of type 316 austenitic stainless steels by EBSD, Materials Characterization 61 (10) (2010) 913-922.

[42] M. Calcagnotto, D. Ponge, E. Demir, D. Raabe, Orientation gradients and geometrically necessary dislocations in ultrafine grained dual phase steels studied by 2D and 3D EBSD, Materials Science and Engineering A 527 (2010) 2738-2746.

[43] T. Britton, H. Liang, F. Dunne, A. Wilkinson, The effect of crystal orientation on the indentation response of commercially pure titanium: experiments and simulations, Proceedings of the Royal Society of London A: Mathematical, Physical and Engineering Sciences 466 (2115) (2010) 695-719.

[44] T. Ruggles, D. Fullwood, J. Kysar, Resolving geometrically necessary dislocation density onto individual dislocation types using ebsd-based continuum dislocation microscopy, International Journal of Plasticity 76 (2016) $231-243$.

[45] A. Arsenlis, D. M. Parks, Crystallographic aspects of geometrically-necessary and statistically-stored dislocation density, Acta Materialia 47 (5) (1999) 1597-1611.

[46] J. Wheeler, E. Mariani, S. Piavzolo, D. Prior, P. Trimby, M. Drury, The weighted Burgers vector: a new quantity for constraining dislocation densities and types using electron backscatter diffraction on 2D sections through crystalline materials, Jour- 
nal of Microscopy 233 (2009) 482-494.

[47] L. Kubin, A. Mortenson, Geometrically necessary dislocations and strain-gradient plasticity: a few critical issues, Scripta Materialia 48 (2003) 119-125.

[48] I. Groma, X-ray line broadening due to an inhomogeneous dislocation distribution, Physical Review B 57 (1998) 7535-7542. 